\title{
The Laser Inter-Laminar Reinforcement of Continuous Glass Fiber Composites
}

\author{
Huade Tan (Corresponding Author) \\ Graduate Research Assistant \\ Email: ht2288@columbia.edu \\ Phone: 646-691-0720 \\ Fax: 212-666-2393 \\ Gen Satoh \\ Email: gs2358@columbia.edu \\ Y. Lawrence Yao \\ Email: yly1@columbia.edu \\ Manufacturing Research Laboratory \\ Department of Mechanical Engineering \\ Columbia University \\ 500 West $120^{\text {th }}$ st Mudd bldg, rm 220 \\ New York, New York, 10027
}

Inter-laminar crack initiation and propagation is a major mode of failure in laminate fiber

reinforced composites. A laser reinforcement process is developed to bond layers of glass fabric prior to the vacuum assisted transfer molding of laminate composites. Glass fabric layers are bonded by fusing a dense glass bead to fibers within the laser focal volume, forming a 3D reinforcement architecture. Coupled heat transfer and viscous flow modeling is used to capture the temperature and morphology evolution of glass during the reinforcement process under experimentally observed conditions. Mode I double cantilever beam testing is performed to quantify the effects of laser inter-laminar reinforcements on composite delamination resistance. Post mortem high resolution imaging of the fracture surface is used to characterize the toughening mechanism of the inter-laminar reinforcements. Improved delamination resistance of laser reinforced composites derives from crack arrest and deflection mechanisms, showing a positive correlation to the reinforcement thickness.

Keywords: A. Glass fibres; B. Delamination; D. Mechanical testing; E. Preform 


\section{$1 \quad$ Introduction}

Laminate polymer matrix composite fabrication consists of the lay-up and consolidation of either pre-impregnated (prepreg) or stitched / woven fabric (preform) fiber reinforcements. Due to the prepreg lay-up process, all reinforcement fibers are aligned in plane with no fiber alignment in the thickness direction. Prepreg laminates exhibit high fiber packing fractions and high strength along the fiber directions, but poor through thickness strength and fracture toughness. The insertion of reinforcing pins between layers of prepreg prior to curing, Zpinning, has been shown to yield greater delamination resistance at the cost of planar strength and stiffness [1]. Z-pins displace fibers in the lamina and introduce pockets of resin rich regions in an otherwise densely packed planar fiber architecture [2].

Fiber placement and resin infusion processing dictate the final form and reinforcement architecture of a composite part. Reinforcement architecture is the determining factor in composite mechanical properties. The ideal reinforcement architecture of a composite subject to multi axial loads is often three dimensional. This work develops a novel manufacturing process in the fabrication of preform laminate composites to improve inter-laminar strength and to allow greater flexibility in their design and application.

Preform laminate fabrication benefits from lower handling costs (no need to refrigerate fabrics after matrix impregnation) and lower tooling costs (out of autoclave processing) over prepreg processing $[3,4]$. In conjunction with liquid resin transfer molding processes, stitched and woven fiber preforms with multi directional fiber architectures are an attractive alternative to prepregs. Bilisik and Bogdanovich investigated weaving and stitching techniques to manufacture composites with three dimensional fiber reinforcement preforms, which showed improved the fracture toughness and through thickness strength $[5,6]$. The introduction of woven or stitched fibers in the thickness direction displaces fibers in the planar directions similar 
to Z-pinning. Such 3D woven preforms exhibit greater out of plane strength and toughness but lower fiber volume fraction and in-plane strength than similar 2D laminates [7].

Laser joining of fibrous materials employs a direct fusion process, unlike mechanical or chemical bonding methods, which offers better seam quality and strength compared to stitching or weaving and has the potential to be applied in the fabrication of $3 \mathrm{D}$ reinforced preforms. The use of near infrared lasers to join thermoplastic fabrics has previously been explored for textile applications such as air bags, medical fabrics, and protective garments that require high strength, high precision, and seamless joints [8,9]. In these applications, the added cost of laser processing is justified by high weld rates, localized joints, watertight sealing, and reduced handling costs. Unlike stitching or weaving, laser processing of fabrics is a non contact process and does not mechanically disrupt the nearby fiber architecture [10]. Laser inter-laminar reinforcement offers the potential for high reinforcement density and direct fiber to fiber bonding in the $\mathrm{Z}$ direction, while allowing reinforcements to be restricted to localized regions, i.e. near existing stress concentrations.

As observed in textile joining investigations, a challenge in the fusion reinforcement of woven fibers is the undesirable formation of voids and discontinuities in the processing zone $[8,9]$. Woven textiles and composite preforms are composed of many fiber bundles, each consisting of hundreds of fibers. Flow and densification mechanisms induce local strains at the interface between the melt and the fiber preform because the relative density of the reinforcement is much greater than the initial material, as is common in sintering processes [11]. During laser fusion processing, densification and surface tension drives the melt to flow away from the center of the laser spot toward the low density regions of the unprocessed fiber bundle, which forms voids within the process volume and leads to poor reinforcement strength. In order to achieve fully dense inter laminar reinforcements, we investigate the physical challenges posed by the densification and flow behaviors of the fiber preform during the fusion process. 
One solution to the relative density and void formation problem in the laser joining of thermoplastic fabrics is to introduce a filler material into the process volume. These filler materials used consist of additive modified polymers that selectively absorb near infrared radiation $(1064 \mathrm{~nm})$, originally developed for the transmission welding of thermoplastics [8]. No selectively absorbing filler material exists for the laser joining of glass. The addition of bulk soda lime glass is investigated as a filler material for the laser processing of glass fabrics. Soda lime glass is highly transmitting at near infrared $(1064 \mathrm{~nm})$ radiation and does not contribute to fiber preform heating during laser processing. An alternate absorption mechanism is found to take place within the fiber preform without a selectively absorbing filler material.

This work develops an inter-laminar laser reinforcement process for glass fiber preforms, overcoming the densification and flow induced void formation problems inherent in the laser fusion bonding of fabrics. The novel process produces fully dense glass reinforcements by the co-fusion of a soda lime glass bead to planar fabric fibers at the laser focal volume. Heating is accomplished through the multiple scattering assisted absorption of laser energy within the fiber preform without a preferentially absorbing filler material. A two phase, temperature dependent flow model is used to simulate the melt pattern and reinforcement morphology observed during the laser process. The effects of laser inter-laminar reinforcements on delamination resistance are characterized through Mode 1 double cantilever beam delamination testing. Scanning electron microscopy (SEM) imaging of double cantilever beam fracture surfaces is used to investigate how laser inter-laminar reinforcements affect the propagation of a delamination crack. The development of this novel inter-laminar reinforcement method offers a new approach for fabricating 3D reinforcement architectures within a preform laminate composite.

\section{Experimental Setup}

Laser reinforcement of a plain woven E-glass fiber epoxy laminate composite is performed during the fiber preform lay-up process prior to liquid resin infusion. A dense inter- 
laminar reinforcement is formed by irradiating the fabric preform with a focused laser source and fusion bonding a soda-lime glass bead between the glass fabric layers. Laser processed glass fiber preforms are fabricated into laminate panels by a liquid resin infusion process using vacuum assisted resin transfer molding (VARTM). Panels were cut into double cantilever beam specimens following the ASTM standard D5528 for delamination resistance testing, as depicted in Fig.1. Inter laminar reinforcements bonded across four and eight glass fabric layers were fabricated and tested using this method. SEM imaging of reinforcement and fiber fracture surfaces is performed to further characterize the crack propagation process during Mode I delamination.

\subsection{Through Thickness Laser Reinforcement}

Laser fusion processing of E-glass fibers was achieved using an Nd:YAG laser from GSI Luminotics operating at 30W. Glass reinforcements consist of 99.98\% E-glass fibers with no surface coating prepared from $136 \mathrm{~g} / \mathrm{m}^{2}$ plain woven fabric with 7 fiber bundles per centimeter and 300 fibers per bundle. Inter laminar reinforcements were formed by the introduction and co-fusion of $1 \mathrm{~mm}$ diameter soda lime glass beads between the glass fabric layers using a focused laser source. In order to limit the diameter of the fusion zone to the diameter of the fill bead, the laser was focused to a spot size of $0.8 \mathrm{~mm}$ using a final objective with a numerical aperture of 0.26. Fabric placement and motion control was accomplished using a Staubli six axis robotic manipulator with a flat plate sample holder. A schematic for the laser irradiation of a glass fiber preform and the resulting melt morphology is depicted in Fig. 2A.

Bulk E-glass and soda lime glass are both highly transmitting at near infrared (1064 nm) radiation wavelengths. Without the addition of a preferentially absorbing filler material, an alternate means of laser energy absorption is needed to facilitate fusion bonding. This study found that densely packed E-glass fiber fabrics are strongly scattering and of sufficient opacity to absorb laser energy and melt the glass fibers. Investigations of radiation transmission through 
densely packed beds of glass spheres [12] and highly transmitting porous media [13] have similarly reported significant absorption due to multiple surface scattering. Multiple scattering induced absorption of the laser is achieved through the increased mean free path of light traveling through the glass fibers. This mechanism is highly dependent upon the structure and packing density of the fiber preform but was found to be controllable during laser processing.

Although other laser textile applications have evaluated the use of absorptive coatings and filler materials for targeted heating, it was determined from initial irradiation trials that surface coatings induce uneven absorption, which causes rapid uncontrollable vaporization in the sample and leads to retained porosity within the reinforcement. Packed beds of glass fibers with surfacing agents rapidly vaporize due to localized heating at the fiber surface and the dramatic increase in the absorption of infrared radiation at elevated glass temperatures [14]. The temperature of the sample becomes uncontrollable upon glass vaporization and plasma formation, resulting in the undesirable formation of voids within the focal volume. Laser heating by scattering induced absorption is desired for the fusion processing of glass fibers in order to avoid peak temperatures within the laser focal volume.

During laser processing, surface tension induced viscous flow is the dominant material transport mechanism for the fusion and densification of glass fiber preforms, as is consistent in the sintering of glass powders [15,16]. A major challenge to the fusion processing of fibrous systems is the high surface area and low relative density of the initial material, causing glass to flow away from the focal volume during laser processing. The effects of surface tension induced flow of glass fiber systems during laser heating has previously been investigated by Tan et al. [17]. The inter-laminar reinforcement method devised in this work relies on the fusion bonding of a dense bead of soda lime glass between layers of fabric preform to overcome the flow behavior inherent in the fiber system. A schematic of the laser reinforcement method is depicted in Fig. 2B. 


\subsection{Delamination Resistance Measurement}

To measure the effect of laser inter-laminar reinforcements on delamination resistance, double cantilever beam tests are conducted with reinforcements placed on the central axis of the beam at $10 \mathrm{~mm}$ intervals, as depicted in Fig. 1. After laser processing, fiber preforms are infused with system 2000 two part epoxy from FibreGlast industries using a vacuum assisted resin transfer molding method (VARTM) and cured for 24 hours at room temperature. Laminate panels measuring $152 \mathrm{~mm}$ by $200 \mathrm{~mm}$ by 5mm (32 ply) are cut and fabricated into double cantilever beam (DCB) specimens as described by ASTM D5528. A $100 \mu \mathrm{m}$ thick, $50 \mathrm{~mm}$ long polyethylene film is placed at the mid plane of the DCB specimen 10mm ahead of the inter laminar reinforcements to initiate the delamination crack during testing.

Samples are tested under displacement control using an Instron 5948 material testing machine at a constant $5 \mathrm{~mm}$ per minute cross head speed without unloading after crack initiation. Cross head extension is obtained from the Instron linear actuator and load is measured by a $2 \mathrm{kN}$ load cell with $0.01 \mathrm{~N}$ precision. A high resolution synchronized camera (Point Grey GRAS50S5M/C-C) with a 4x objective and data acquisition system was used to capture an image of the crack front at every $1 \mathrm{~mm}$ of cross head displacement while simultaneously recording the extension and load. Mode I fracture energy release rate is calculated using the modified compliance calibration method using the equation

$$
G_{I}=\frac{3 P^{2} C^{\frac{2}{3}}}{2 A_{1} b h^{\prime}}
$$

where $P$ is the load recorded, $b$ is the beam width, $h$ is the beam thickness, $C=\frac{\delta}{P}$ is the compliance of the beam and $A_{1}$ is the slope of the least squares regression of the thickness normalized delamination length as a function of the cubed root of compliance [18].

Load versus displacement and delamination resistance results obtained from DCB tests quantify the fracture toughness along the crack plane. A 1mm cross head displacement interval 
between data points yields sufficient spatial resolution to capture the significantly varying delamination resistance between processed and the non-processed regions along the crack front. Detailed spatial resolution from DCB tests coupled with post mortem imaging of the fracture surface gives added insight into the crack propagation process, allowing for the analysis of delamination propagation in the unreinforced laminate and the effect of crack arrest and deflection at the reinforcements. A group of eight DCB samples were tested for reinforcement thicknesses of 4 and 8 laminae as well as a group of unreinforced laminates. The average delamination resistance, after an initial onset crack length $(\Delta a>5 \mathrm{~mm})$, is calculated for each sample set.

\section{$3 \quad$ Numerical Simulation}

A coupled two dimensional heat transfer and compressible flow model is implemented to simulate the flow behavior of the molten pool during fiber processing. The model concurrently solves the compressible Navier Stokes equations for viscous fluid flow, phase field (Cahn Hilliard) equations for two phase immiscibility and velocity dependent heat equations, as discussed in detail in a previous paper [17]. The numerical model is validated using experimental results of the reinforcement morphology. Simulation results yield greater insight into the temperature dependent behavior of fiber preforms than experiments alone, capturing the combined effects of laser heating, densification, and flow on the resulting reinforcement morphology.

Laser energy is assumed to be absorbed within a volume defined by a Gaussian distribution in the lateral and $\mathrm{z}$ directions. The laser power density is calculated from the total power input into the material over the laser interaction volume. As glass softens, the resistance to flow of the glass fiber preform and glass fill is modeled by the temperature dependent viscosity using the Vogel-Fulcher-Tammann equation: given for E-glass and soda lime glass as [19] 


$$
\eta_{E-\text { glass }}(\mathrm{T})=10^{-4.88+\frac{6227}{T-268}}, \eta_{\text {soda-lime }}(\mathrm{T})=10^{-2.585+\frac{4215}{T-263}}
$$

where $\eta$ is the glass dynamic viscosity $(P a * s)$ and $T$ is the glass temperature in degrees Celsius. The dynamic viscosity of E-glass decreases by eight orders of magnitude in the glass transition range between 700 and $1400^{\circ} \mathrm{C}$. Due to this highly temperature dependent viscosity, glass behaves both as a solid and a liquid during the simulation. The dramatic reduction in glass viscosity dictates much of the physical response of the reinforcement morphology during reinforcement processing.

The glass fiber preform is modeled using the continuous media theory for selective laser sintering developed by Kolossov et al. [20], where the degree of fiber densification is defined by a sintering potential $\pi$ given by

$$
\pi(\boldsymbol{x}, t)=1-e^{-\int_{0}^{t} \zeta(T(\boldsymbol{x}, s)) d s},
$$

where $\pi$ is a continuous variable $[0,1)$ corresponding to either separate fibers at $\pi=0$ or fully dense glass at $\pi=1$. The rate of densification $\zeta(T)$ is first derived by Frenkel and later expanded upon [21-23] to be a temperature dependent function given by

$$
\zeta=\frac{\gamma}{\eta(T) d_{0}}
$$

where $\gamma$ is the surface energy, $\eta(T)$ is the viscosity and $d_{0}$ is a characteristic length scale of the initial material. Neither the surface tension $\left(200-400 \frac{\mathrm{mJ}}{\mathrm{m}^{2}}\right)$ nor characteristic length of glass fiber $(10 \mu m)$ is highly dependent upon temperature. The densification rate of glass fiber may thus be reduced to a function of temperature and viscosity

$$
\zeta(T)=K \frac{1}{\eta(T)}
$$

Viscosity and compaction rates of E-glass and soda lime glass are plotted as a function of temperature in Fig. 3. 
The continuous media approximation of the glass fiber preform defined by Kolossov assumes a homogenous fractional density and thermal conductivity of partially dense glass given by

$$
\begin{aligned}
& \rho=(\alpha+(\beta-\alpha) \pi) \rho_{b u l k}, \\
& k=(a+(b-a) \pi) k_{b u l k},
\end{aligned}
$$

where $\beta=b=1, \alpha=0.4$ defines the initial volume fraction of glass fibers and $\mathrm{a}=0.1$ defines the fractional conductivity between loose fibers and densely packed fibers to the bulk. Bulk thermal conductivity of glass is observed to be relatively constant with respect to temperature in the glass transition range [24]. At sufficient temperatures, glass spontaneously emits and absorbs infrared radiation, leading to a temperature dependent radiation factor in the thermal conductivity of the glass given by

$$
k_{\text {rad }}(\mathrm{T})=\frac{16 n^{2} \sigma T^{3}}{3 \gamma}
$$

where $n$ is the refractive index of glass, $\sigma$ is the Stefan-Boltzmann constant, and $\gamma$ is a geometric parameter relating to the absorption over the mean free path of the material $[25,26]$. Given the anisotropy of the fiber architecture in the planar directions, the material conductivity is assumed to be

$$
k_{i}=\left(a_{i}+\left(b-a_{i}\right) \pi\right) k+k_{r a d}
$$

where $b=1$, and the initial conductivity ratio in the $a_{x}$ and $a_{y}$ directions are assumed to be 0.1 and 0.01 , respectively.

Anisotropic surface tension forces affect the flow of glass at the preform and melt interface caused by the fiber microstructure and glass density gradient at the melt boundary. A density gradient dependent body force is assumed to act on the melt volume given by

$$
\mathbf{F}=\beta \nabla \rho V_{F_{\text {glass }},}
$$


where $\mathbf{F}$ is the densification force, $\beta$ is a constant tuning parameter used to match the morphology of the melt region during processing. The component of $\mathbf{F}$ in the thickness direction is zero given the anisotropic tendency of molten glass to flow along the fiber directions [17].

The model is bounded in a semi-infinite domain with a single horizontal parting line separating the initial volume fraction domains of air and glass fabric preform. Simulations of the laser fusion process include a bead of fully dense glass on the surface of the glass fiber preform. The initial condition of the sintering potential is taken to be $\pi=1$ within the bead and $\pi=0$ everywhere else. No slip and constant temperature boundary conditions are applied at the perimeter of the solution domain. The boundary of the fluid domain is set to zero velocity along the outer boundary of the glass preform and zero pressure along the air boundary such that the total mass of glass is conserved during the simulation.

The set of coupled fluid, phase field, heat and sintering equations are solved concurrently within a spatially resolved finite element domain in a fully coupled backward Euler time integration scheme using COMSOL Multiphysics. The solution domain is discretized with a uniform 2D triangular mesh in order to resolve the air / glass boundary throughout the simulation. Initial and boundary conditions specific for each simulation are described in the results sections 4.1 and 4.2. Key material properties used in this model are listed in Tab. 1.

\section{$4 \quad$ Results and Discussion}

Laser inter-laminar reinforcements are formed by the co-fusion of a soda-lime glass bead at the laser focus volume within a glass fiber preform. The glass bead acts as a filler to overcome the densification and flow effects during localized heating of woven glass fabrics. Reinforcement morphology and penetration is measured experimentally, and a numerical model is implemented to study the physical effects of viscous densification during the laser process. Strength and fracture toughness enhancement of the laser processed composite is measured 
experimentally from double cantilever testing of woven fabric composites reinforced in the mid plane. Delamination resistance is found to be dependent upon the reinforcement thickness.

\subsection{Preform Densification}

Laser irradiation of tightly woven glass fibers causes rapid consolidation of glass within the laser focal volume. Fibers approaching the softening temperature of E-glass shorten and agglomerate to its nearest neighbor, forming pockets of dense glass. Anisotropic shrinkage and wetting of the molten glass into the surrounding fiber materials causes the material at the center of the focal volume to flow outward. The resulting morphology of the melt is a ring of solid glass around the focal volume, as depicted in Figs. 2A and 4.

The melt formation and separation behavior is quantified from morphology

measurements from optical microscopy images of laser irradiated fiber fabrics (Fig. 4). The melt diameter and melt depth are plotted with respect to time in Fig. 5. During the glass densification process, liquid glass flows and expands in both diameter and depth as a function of exposure time. The diameter of the melt pool increases rapidly and approaches a maximum of approximately 1mm, slightly larger than the laser spot size. The depth of the melt pool increases linearly, with no apparent maximum within the range of exposure times evaluated in this study. The flow and separation behavior of the glass melt is characteristic of the laser energy input and densification of the fiber substrate.

Laser energy absorption is highly dependent upon the effect of multiple scattering on the mean free path taken by the beam through the glass fiber preform. Melt flow is highly dependent upon the surface energy and viscosity of the preform. Highly localized heating within the laser focus volume induces large temperature and viscosity gradients within the fibers, which causes preferential flow along the fiber directions. The resulting morphology of the melt is a function of the laser energy profile. As low viscosity glass fibers compact and flow away from the center of the laser focus, incoming laser energy penetrates deeper into the preform and is no longer 
absorbed in that fabric layer. At a sufficient melt diameter, a self limiting state exists such that the fiber surface tension balances the viscous flow resistance and a quasi-static equilibrium is reached in the radial direction.

As molten glass flows away from the focal point, laser radiation passes deeper into the material and the process is repeated in each successive layer. Transmission of the laser energy into the preform occurs linearly in time during the melt separation process as is observed by the linear trend in melt depth depicted in Fig. 5. As glass melts and separates in the layer above, the layer below is exposed to more laser radiation and begins to melt before the maximum diameter is reached in the layer above. Given a collimated laser beam with low dispersion, the melt morphology of the fabric is expected to be constant in diameter with respect to depth. A focused laser beam was used in this study with a numerical aperture of 0.26 , causing the beam profile to diverge away from focus. Beam divergence reduces the beam intensity away from the focal plane, increasing the melt diameter and limiting the reinforcement thickness. This effect was not observed in the range of preform thicknesses evaluated.

Coupled laser heating, densification, and flow observed during experimental trials are captured in the transient multi physics numerical simulation, with a Gaussian heat source input into a continuous substrate. As depicted in Fig. 6, the simulation domain is broken up into two halves with a mid plane boundary between air and the partially dense glass fabric preform. Edges of the preform are set to a ninety degree contact angle with the domain boundary. The domain boundary is set to room temperature throughout the simulation and is allowed to have non zero velocities at the boundaries with air. The glass sintering potential, density, and temperature are solved concurrently and resolved in space and time within the computation domain. The temperature and density distribution of the glass fiber preform is depicted in snap shots of the simulation domain in Fig. 6. The effects of bundle densification and flow dynamics on the morphology of the melt pool during laser processing are plotted as a function of time in 
Fig. 7. Simulation results of the melt diameter and depth are in good agreement with the experimental results plotted in Fig. 5.

The peak melt temperature from the numerical simulation is observed to increase rapidly early in the simulation and reaches a maximum as the laser absorption, heat accumulation and flow effects reach a quasi-steady state as depicted in Fig 7. The melt morphology is driven by the temperature field of the solution, as dictated by the temperature dependent viscosity and sintering potential. For a stationary laser heat source, the temperature and diameter of the melt reaches a maximum, as is confirmed in experiments. Morphology agreement between the simulation and experiments suggest that this model is well suited for studying the laser reinforcement process.

\subsection{Reinforcement Morphology}

A dense reinforcement between multiple fabric layers is formed when laser radiation is transmitted into a glass fiber fabric through a solid fill material. Solid glass fill in the form of soda lime glass beads serve two functions in the reinforcement process: to facilitate laser energy transmission into the fabric and to replace the fabric material as it flows away from the laser heat source. During the laser reinforcement process, the fiber preform absorbs focused laser energy and conducts heat back into the soda lime bead. Optical microscopy images of laser interlaminar reinforcements show a dense transparent core of fill material surrounded by a dense shell of fused fiber, as depicted in Figs. 8 and 9. The interface between the fill fiber materials is visible under optical microscopy because the index of refraction of soda lime glass (1.52) and Eglass (1.56) is sufficiently different, as shown in Fig. 9.

Similar to the irradiation of glass fiber preforms, laser absorption is caused by the multiple scattering of near infrared radiation in focal volume beneath the fill bead. Multiple scattering induced absorption of glass diminishes as fibers compact into a dense reinforcement. As fibers coalesce and the number of scattering surfaces is reduced, laser radiation is transmitted 
deeper into the preform. Thus, laser absorption during glass reinforcement processing is a self limiting process. As the glass melt penetrates through all layers of fabric, the laser energy absorbed by the preform is reduced, and a quasi-steady state condition is achieved when the effects of heat conduction, laser absorption and viscous flow are balanced. The densification and flow of the fabric preform allows for the addition of glass fill into the process volume, bonding with the initially low volume fraction glass fibers. As the fill material penetrates deeper into the preform, laser energy is transmitted further into the fiber fabric. The final morphology of the reinforcement is limited by the initial geometry of the fill material.

A spherical soda lime bead with a 1mm diameter, as used in this study, is sufficient to form a continuous reinforcement of up to eight fabric layers in depth (approximately $1.2 \mathrm{~mm}$ ). Laser processing of fewer than 8 layers of fabric may be formed with this process, resulting in a slightly larger reinforcement diameter. Processing in excess of eight fabric layers showed incomplete melt penetration and limited fiber connectivity. Preform fiber packing density played an important role in the penetration process, requiring mechanical pressure to be applied on the fabric in order to ensure fill penetration through multiple layers. Reinforcement cross sections (Fig. 9) show that the reinforcement diameter remains relatively constant throughout its thickness and fiber connectivity is preserved in each layer of the fabric.

Numerical simulations of the laser inter-laminar reinforcement process are carried out with the same modeling scheme as the preform densification process. The simulation domain is split between air and glass fiber preform in the same manner as discussed in section 4.1 with the exception of a $1 \mathrm{~mm}$ diameter bead of fully dense glass above the fibers. The laser source is approximated with a Gaussian heat input at the interface between the glass bead and the perform surface. The morphology and temperature evolution during the fill induced laser reinforcement process are plotted in Figs. 10 and 11. The melt morphology obtained from simulations in Fig. 10 captures the reinforcement structure shown in Fig 9. Similar to the preform densification 
behavior, the reinforcement penetration behaves linearly in time while the reinforcement diameter increases rapidly early in the process. The diameter of the reinforcement continues to increase throughout the reinforcement process, unlike the behavior of the fiber preform without the presence of the filler material.

A maximum temperature of the melt, plotted in Fig.11, is quickly reached in the laser reinforcement process. This state of thermal equilibrium is achieved during reinforcement processing due to the self limiting laser absorption and densification behaviors of the glass fiber preform. The maximum temperature of the melt pool is consistent with the softening range of Eglass. Time dependent fiber densification and laser absorption effects captured in this model are shown to yield good morphological agreement with experimental results. From the agreement between numerical and experimental results, it is determined that the dynamics captured by the numerical model are the controlling mechanisms in the laser reinforcement process.

\subsection{Delamination Resistance}

Evaluation of laser inter-laminar reinforcement effects on mode 1 delamination resistance has been carried out using double cantilever beam (DCB) bending tests of plain woven glass fiber composite samples. While delamination is observed to propagate smoothly along the mid plane of unreinforced glass composite beams, reinforced samples exhibit observable start, stop crack propagation behavior during delamination testing with rapid crack propagation between each reinforcement and periods of crack arrest at the reinforcements. The characteristic start stop crack propagation behavior is observed in the discontinuous load versus extension results shown in Fig. 12. Characteristic delamination resistance measurements of laminate samples with and without laser inter-laminar reinforcements are plotted in Fig. 13. The discontinuous loading curve observed in laser reinforced samples differs dramatically from the relatively smooth fracture process observed in unreinforced samples, also plotted in Fig. 13. 
During DCB testing, crack arrest at length intervals consistent with the reinforcement spacing suggests that delamination is inhibited by the laser reinforcement. Dense glass reinforcements formed between fabric plies is of sufficient strength to resist the continuous propagation of the delamination crack along the fiber / matrix interface. During periods of crack arrest, the double cantilever beam is strained until sufficient stresses develop within the beam to propagate the crack around the reinforcement by fracturing the bonded fibers. As a result of the higher stresses stored in the reinforced DCB sample, crack deflection and branching across lamina is often observed at laser reinforcements. Evidence of crack deflection and fiber fracture at laser reinforcements are captured in SEM images of the reinforcement fracture surface presented in section 4.4 .

Average fracture energies calculated from repeated DCB tests are plotted in Fig. 14. It is observed in this figure that there is a positive trend between reinforcement thickness and fracture energy. Because fibers are bonded to a continuous reinforcement between lamina, the mechanism of laser inter-laminar toughening is one of fiber and reinforcement fracture. Delamination resistance is thus a function of the number of fibers bonded to the reinforcement and the fracture strength of the reinforcement itself. Planar reinforcement density is held constant in all the samples tested. Laser processed samples overall exhibit higher fracture energies than non-reinforced samples. Higher peak fracture energies observed at each reinforcement location, in Fig. 13, suggests that the average fracture energy of the sample is dependent upon the number of reinforcements in plane.

The increase in average delamination resistance observed in the laser reinforced samples is comparable with other mechanically reinforced composites. Unidirectional glass-fiber composites typically exhibit inter-laminar fracture toughness values of around 0.6 to $0.8 \mathrm{~kJ} / \mathrm{m}^{2}$. Mouritz et al. reported that orthogonal 3D woven fabrics exhibit double the delamination resistance $\left(1.4 \mathrm{~kJ} / \mathrm{m}^{2}\right)$ of traditional fiberglass composites with a reduction in fiber volume 
fraction from 0.59 to 0.37 [27]. The $2 \mathrm{D}$ plane weave glass fiber composite used in the current study exhibits higher baseline fracture toughness $\left(0.9 \mathrm{~kJ} / \mathrm{m}^{2}\right)$ with a lower initial fiber volume fraction (0.42). Laser reinforced samples with similar fiber volume fractions exhibit a 50\% increase in average fracture toughness (about $1.3 \mathrm{~kJ} / \mathrm{m}^{2}$ ) with minimal disruption to the global fiber architecture. While Koh et al. reports a 200 to 500\% increase in delamination resistance when 0.5 to $4 \%$ z-pins by volume are introduced in a unidirectional carbon fiber composite [28], a direct comparison between laser reinforcement and Z-pinning is not available due to the predominant application of Z-pins in carbon/graphite fiber prepregs rather than glass fiber preform composites. Further work is needed to evaluate the effect of planar reinforcement density on delamination resistance using the current laser process.

\subsection{Fracture Surface Morphology}

Contrasting fracture surfaces between unreinforced and reinforced laminate fracture surfaces are depicted in Figs.15 and 16. Delamination of the unreinforced glass preform composite takes place at the fiber/matrix interface, leaving an undulating surface profile defined by the woven fiber structure. In these regions, only the characteristic brittle fracture of the matrix material is observed with little to no fiber fracture. In contrast to the un-reinforced regions, the fracture surface at laser reinforcements is characterized by significant fiber fracture and out of plane crack propagation. Fig. 16 illustrates that crack deflection and fiber fracture effectively lead to positive and negative features on the opposing sample fracture surfaces. These features remain from intact inter-laminar reinforcements after the significant crack deflection and fiber fracture around the reinforcement. The effect of crack deflection is observed in the branching of cracks to adjacent layers of fabric during DCB testing. SEM images of the reinforcement surface after testing show a high density of fibers fractured at the surface of the exposed reinforcements (Fig. 17). Fiber fracture and crack deflection are two key mechanisms for the increase in fracture toughness observed in laser reinforced samples. 
As observed during testing, rapid delamination through unreinforced laminate regions

tended to occur after elastic energy is released from a highly stressed region in the beam. Rapid crack propagation sometimes resulted in the failure of the next reinforcement. Reinforcement failure is characterized by the brittle fracture of the solid glass with little to no fiber fracture or out of plane crack deflection. Reinforcement failure is more pronounced in the eight layer reinforcement group due to the higher stresses and peak fracture energies achieved at the reinforcement. The tendency for eight layer reinforcements to fracture is observed in the wider distribution of the average fracture energy results presented in Fig. 14.

\section{$5 \quad$ Conclusions}

A method for the laser reinforcement of glass fabric preforms has been developed to selectively bond glass fiber composites in the laminate thickness direction. The application of laser fusion processing has been evaluated on glass fabrics used in the vacuum assisted resin transfer molding of an epoxy polymer matrix composite. Melt and reinforcement formation dynamics have been simulated using a multi physics numerical method to model the viscous flow, densification, and heating effects of glass fiber preforms during the laser fusion process. Numerical simulations of reinforcement diameter and depth have been validated with measurements of fabric melt morphology observed under optical microscopy.

Mechanical testing of laser reinforced samples has shown an increase in Mode I fracture toughness as a function of the reinforcement thickness. Toughening effects of inter laminar reinforcements is observed in both load versus extension and delamination resistance curves. Scanning electron microscopy images of the fracture surface show significant fiber fracture and crack deflection at the reinforcements, corresponding to the experimentally observed increase in local fracture toughness. Further testing is required to determine the effects of laser interlaminar reinforcements on the laminate’s planar mechanical properties. 
Delamination resistance increases in proportion to the laser inter-laminar reinforcement thickness up to eight laminae. Further work is necessary to evaluate ways to increase the maximum laser reinforcement thickness. The current process is limited by the geometry and insertion method of the soda lime fill material. Fill geometries with higher aspect ratios may be evaluated as a means to form inter laminar reinforcements in excess of eight fabric layers.

A novel laser inter-laminar reinforcement process has been shown to be a viable method for the fabrication of 3D reinforced preforms to enhance the delamination resistance of woven glass fiber composites. The effects of laser fusion on fiber architecture and morphology have been examined through experiments and numerical simulations. Mode I fracture toughness findings show an increased delamination resistance as a function of reinforcement thickness and in plane density. Possible efforts to further improve Mode I fracture toughness with this method has been discussed. The application of laser inter-laminar reinforcements in areas of high delamination stress concentrations presents a flexible and effective method to mitigate the onset of delamination in preform fabricated composites.

\section{Acknowledgements}

The use of material testing equipment and camera system was provided by Professor Kristin Meyers with help from Ms. Kyoko Yoshida. Partial financial support from Columbia University is gratefully acknowledged. 


\section{References}

[1] Byrd L, Birman V. Effectiveness of z-pins in preventing delamination of co-cured composite joints on the example of a double cantilever test. Composites Part B: Engineering 2006;37:365-78.

[2] Huang $\mathrm{H}$, Waas AM. Compressive response of Z-pinned woven glass fiber textile composite laminates: Modeling and computations. Composites Science and Technology 2009;69:2338-44.

[3] Li W. Process and Performance Evaluation of the Vacuum-Assisted Process. Journal of Composite Materials 2004;38:1803-14.

[4] Niggemann C, Gillespie JW, Heider D. Experimental Investigation of the Controlled Atmospheric Pressure Resin Infusion (CAPRI) Process. Journal of Composite Materials 2008;42:1049-61.

[5] Bilisik K. Multiaxis 3d woven preform and properties of multaxis $3 d$ woven and $3 d$ orthogonal woven carbon/epoxy composites. Journal of Reinforced Plastics and Composites 2009;29:1173-86.

[6] Bogdanovich AE. Advancements in manufacturing and applications of 3-d woven preforms and composites. 16th international conference on composite materials, 2006, p. 1-10.

[7] Mohamed $\mathrm{MH}$, Wetzel KK. 3D woven carbon/glass hybrid spar cap for wind turbine rotor blade. Journal of Solar Energy Engineering 2006;128:562.

[8] Jones I. Improving productivity and quality with laser seaming of fabrics. Technical Textiles International 2005:35-8.

[9] Niebel V, Weinert N, Gries T, Seliger G. Technology for overlap-free joining of semifinished textile products. Technical Textiles 2009;52:E68-69.

[10] Binetruy C, Clement S, Deleglise M, Franz C, Knapp W, Oumarou M, et al. Glue-free assembly of glass fiber reinforced thermoplastics using laser light. SPIE, vol. 8065, 2011, p. 1-7.

[11] Rahaman MN, De Jonghe LC, Scherer GW, Brook RJ. Creep and Densification During Sintering of Glass Powder Compacts. Journal of the American Ceramic Society 1987;70:766-74.

[12] Chen JC, Churchill SW. Radiant heat transfer in packed beds. AIChE Journal 1963;9:35-41.

[13] Sun W, Loeb NG, Fu Q. Finite-difference time-domain solution of light scattering and absorption by particles in an absorbing medium. Applied Optics 2002;41:5728-43. 
[14] Dvurechensky A V., Petrov VA, Reznik VY. Spectral emissivity and absorption coefficient of silica glass at extremely high temperatures in the semitransparent region. Infrared Physics 1979;19:465-9.

[15] Kuczynski GC. Study of the sintering of glass. Journal of Applied Physics 1949;20:1160-3.

[16] Kingery WD, Berg M. Study of the Initial Stages of Sintering Solids by Viscous Flow, Evaporation-Condensation, and Self-Diffusion. Journal of Applied Physics 1955;26:120512.

[17] Tan H, Yao YL. Laser Joining of Continuous Glass Fiber Composite Preforms. Journal of Manufacturing Science and Engineering 2013;135.

[18] Standard Test Method for Mode I Interlaminar Fracture Toughness of Unidirectional Fiber-Reinforced Polymer Matrix Composites. Annual Book of ASTM Standards, vol. 01, 2007, p. 1-12.

[19] Fluegel A. Glass viscosity calculation based on a global statistical modelling approach. Glass Technology: European Journal of Glass Science And Technology Part A 2007;48:1330.

[20] Kolossov S, Boillat E, Glardon R, Fischer P, Locher M. 3D FE simulation for temperature evolution in the selective laser sintering process. International Journal of Machine Tools and Manufacture 2004;44:117-23.

[21] Hopper RW. Coalescence of Two Viscous Cylinders by Capillarity: Part 1, Theory. J Am Ceram Soc 1993;76:2947-52.

[22] Scherer GW. cell models for viscous sintering. Journal of the American Ceramic Society 1991;74:1523-31.

[23] Mackenzie JK, Shuttleworth R. A Phenomenological Theory of Sintering. Proceedings of the Physical Society Section B 1949;62:833-52.

[24] Tempel L Van Der. Thermal Conductivity of a Glass: II . The Empirical Model. Glass Physics and Chemistry 2002;28:147-52.

[25] Grove FJ. Spectral Transmission of Glass at High Temperatures and Its Application to Heat-Transfer Problems. Journal of the American Ceramic Society 1961;44:1956-9.

[26] Condon EU. Radiative Transport in Hot Glass. Journal of Quantitative Spectroscopy and Radiative Transfer 1968;8:369-85.

[27] Mouritz AP, Baini C, Herszberg I. Mode I interlaminar fracture toughness properties of advanced textile fibreglass composites. Glass 1999;30:859-70. 
[28] Koh TM, Feih S, Mouritz a. P. Experimental determination of the structural properties and strengthening mechanisms of z-pinned composite T-joints. Composite Structures 2011;93:2222-30. 


\section{FIGURES}

Fig. 1: A) Sample schematic of a laser joined DCB specimen. B) An image of a DCB test during displacement controlled loading. Synchronized capture of high resolution DCB fracture images enables the calculation of fracture energy with high spatial resolution.

Fig. 2: Inter laminar laser reinforcement process schematic showing $A$ ) the irradiation of a stack of glass fabric resulting in the formation of a void in through the material with a dense ring of glass around the laser spot. B) The through thickness laser reinforcement process employs a bead of dense glass fill to bond the glass melt, forming a dense reinforcement through the initial stack of glass fabrics.

Fig. 3: Temperature dependent glass viscosity from VFT model and densification rates of Soda Lime and E-glass as obtained from global glass composition calculations [19].

Fig. 4: Optical micrograph of the surface fiber morphology after laser irradiation of a single layer of plain woven fabric. A dense ring of glass is formed during the heat induced compaction of fibers within the fabric.

Fig. 5: Measurements of the of the melt pool morphology due to laser irradiation on a stack of glass fabrics as a function of time taken from optical microscopy imaging of the fabric after processing. Note that the diameter of the melt ring reaches a steady state and plateaus while the depth of laser penetration is observed to be approximately linear up to the maximum thickness tested.

Fig. 6: Numerical output of the glass densification process without filler material at 0.2 and 2.4 seconds. The substrate is shown to form a void in the center of the laser focus with the maximum temperature at the base of the laser penetration depth.

Fig. 7: Time dependent melt morphology: temperature depth and diameter obtained from numerical simulation. The melt diameter and depth is shown to follow the same trend as the experiments observed under optical microscopy.

Fig. 8: Optical micrograph of a through thickness reinforcement formed through four layers of glass fabric using a dense filler glass. Note that the center of the reinforcement is fully dense with little to no porosity, as observed from the greater transparency than the surrounding fabric.

Fig. 9: Cross section optical micrograph taken of a four layer through thickness joint cross section. Note that the joint is mostly dense with little to no porosity throughout its thickness. The Soda Lime fill glass is observable in optical images from the contrasting index of refraction from the E-glass fiber melt.

Fig. 10: Numerical simulation snap shots of fill induced joining at 0.2 and 2.0 seconds showing the time dependent evolution of the melt morphology during laser fusion joining. Void formation in the substrate is observed to be suppressed by the addition of the fill material. The temperature and morphology of the joint is otherwise shown to be unchanged from the non fill simulation. 
Fig. 11: Time dependent reinforcement morphology output from the numerical simulation, plotting temperature depth and diameter as a function of time. The linear trend of laser penetration observed is consistent with the morphology of the non joined simulation.

Fig. 12: DCB load versus cross head extension output from the Instron output showing higher loading at each characteristic stop in the discontinuous crack propagation corresponding to the reinforcement locations within the sample.

Fig. 13: DCB fracture energy showing significantly higher fracture energy at reinforcement locations due to the increase in load as observed in the load versus extension output. Greater fracture energy is achieved by crack arrest, thereby increasing the load and bending energy required for the crack to propagate around the reinforcement.

Fig.14: Compiled data of the average delamination resistance as a function of the number of fabric layers through which the reinforcement is bonded. Note that the average fracture energy follows an approximately linear trend with the joint thickness due to the higher maximum load and peak fracture energies exhibited at the joint locations. Eight samples were tested at each condition. Error bars signify the standard deviation between tests.

Fig. 15: SEM images of the delamination fracture surface between two layers of plain woven glass fabric composite. The crack front is observed to neatly propagate along the surface of the lamina between fabric layers, choosing the path of least resistance.

Fig. 16: SEM images of the fracture surfaces around a laser reinforced section showing significant fiber fracture and crack branching behavior due to crack arrest and deflection at joints. The A) positive and B) negative imprint of a reinforcement remains on the surface of the DCB sample after the crack has propagated around it. Note that the depth of the fracture surface in the fabric material corresponds to half of the reinforcement thickness. Multiple layers of fabric are exposed on the fracture surface.

Fig. 17: SEM image of fiber fracture surfaces around a single reinforcement. Fibers from multiple layers are observed from the cross ply orientations of the fracture surfaces. 
TABLES

Tab. 1: Physical properties of glass and air used in processing simulations.

\begin{tabular}{ccccc}
\hline parameter & units & E-glass & Soda Lime & value (air) \\
\hline$\rho$ & $\mathrm{kg} / \mathrm{m}^{3}$ & 2540 & 2580 & 1 \\
\hline$C p$ & $\mathrm{~kJ} / \mathrm{kg} \mathrm{K}$ & 0.84 & 0.81 & 1 \\
\hline$\eta$ & $\mathrm{Pa} \cdot \mathrm{s}$ & eq. (2) & eq. (2) & $<<\eta_{\text {glass }}$ \\
\hline$k_{\text {bulk }}$ & $\mathrm{W} / \mathrm{m} \mathrm{K}$ & 1.35 & 1.3 & 0.03 \\
\hline$\sigma$ & $\mathrm{N} / \mathrm{m}$ & & 0.31 & \\
\hline
\end{tabular}

